\title{
Low-Molecular-Weight Heparin and Protamine-Based Polyelectrolyte Nano Complexes for Protein Delivery (A Review Article)
}

\author{
Masayuki Ishihara $^{1^{*}}$, Satoko Kishimoto ${ }^{1,2}$, Megumi Takikawa ${ }^{3}$, Yasutaka Mori ${ }^{1,4}$, Shingo Nakamura ${ }^{5}$, \\ Masanori Fujita ${ }^{1}$ \\ ${ }^{1}$ Research Institute, National Defense Medical College, Tokorozawa, Japan; ${ }^{2}$ Research Fellow of the Japan Society for the Promotion
of Science, Tokyo, Japan; ${ }^{3}$ Department of Plastic Surgery, National Defense Medical College, Tokorozawa, Japan; ${ }^{4}$ Aeromedical
Laboratory, Japan Air Self-Defense Force, Sayama, Japan; ${ }^{5}$ Department of Surgery, National Defense Medical College, Tokoro-
zawa, Japan. \\ E-mail: *ishihara@ndmc.ac.jp
}

Received October $1^{\text {st }}, 2011$; revised November 14 ${ }^{\text {th }}, 2011$; accepted November $28^{\text {th }}, 2011$.

\begin{abstract}
We produced low-molecular-weight heparin/protamine micro (nano) particles (LMW-H/P MPs'NPs) as a carrier for heparin-binding growth factors $(G F s)$, such as fibroblast growth factor $(F G F)-2$ and various GFs in platelet-rich plasma (PRP). A mixture of LMW-H (MW: approximately $5000 \mathrm{Da}, 6.4 \mathrm{mg} / \mathrm{ml})$ and protamine (MW: approximately $3000 \mathrm{Da}, 10 \mathrm{mg} / \mathrm{ml}$ ) at a ratio of 7:3 (vol:vol) yields a dispersion of micro (nano) particles (200 $\mathrm{nm}-3 \mu \mathrm{m}$ in diameter). The diluted LMW-H solution in saline $(0.32 \mathrm{mg} / \mathrm{ml})$ mixed with diluted protamine $(0.5 \mathrm{mg} / \mathrm{ml})$ at a ratio at 7:3 (vol:vol) resulted in soluble nanoparticles (approximately $100 \mathrm{~nm}$ in diameter). The generated NPs could be then stabilized by adding $2 \mathrm{mg} / \mathrm{ml}$ dextran $(M W: 178-217 \mathrm{kDa}$ ) and remained soluble after lyophilization of dialyzed LMW-H/P NPs solution. The LMW-H/P MPs.NPs adsorb GFs, control their release, protect GFs and activate their biological activities. Furthermore, administration of GFs-containing F/P MPs.NPs exhibited significantly higher inductions of vascularization and fibrous tissue formation in vivo than GFs alone. LMW-H/P MPs.NPs can also efficiently bind to tissue culture plates and retain the binding of GFs. The LMW-H/P MPs.NP-coated matrix with various GFs or cytokines provided novel biomaterials that could control cellular activity such as proliferation and differentiation. Thus, $L M W-H / P$ $M P s \cdot N P s$ are an excellent carrier for GFs and are a functional coating matrix for various kinds of cell cultures.
\end{abstract}

Keywords: Polyelectrolyte Complexes, Nanoparticles, Heparin-Binding Growth Factors, Platelet-Rich Plasma, Drug Delivery

\section{Introduction}

Polyelectrolyte complexes (PECs) are produced by electrostatic interactions between oppositely charged polyelectrolytes. When this interaction occurs at non-equivalent ratios, nonstoichiometric PECs are produced, causing each PECs to carry an excess charge. Proteins interact with both synthetic and natural PECs [1,2]. These binding characteristics, along with a simple preparation, allow PECs to be an excellent model for studying the in vivo behavior of charged biopolymers as well as having potential applications in medicine and biotechnology [3]. Reported data indicate that polyanions and polycations can bind to proteins below and above their isoelectric points, respectively. These interactions can result in so- luble complexes, complex coacervation and/or the formation of amorphous precipitates [1,2]. Main aspects studied by different authors are compositions of PECs obtained under various experimental conditions, such as the strength and position of ionic sites, charge density, and rigidity of polymer chains as well as chemical properties such as solubility, $\mathrm{pH}$, temperature, and concentration [3].

Those electrostatic interactions are also important because of their similarity to biological systems [4]. Interactions between proteins and nucleic acids, for example, play a role in the transcription process [1]. DNA/chitosan PECs [5], chitosan/chondroitin sulfate PECs and chitosan/hyaluronate PECs [6] were described as gene and drug micro-carriers. Moreover, PECs that are insoluble 
also have potential applications as membranes, microcapsules, micro (nano) particles, and scaffolds for tissue engineering [7].

Basic protamine molecules complexed with acidic molecules such as heparin form a microcomplexes through ionic interactions. We previously have reported the a low-molecular-weight heparin/protamine micro (nano) particles (LMW-H/P MPs-NPs) which we originally prepared as PECs $[8,9]$. LMW-H/P MPs-NPs are specifically bound to FGF-2 and other various heparin-binding growth factors (GFs) in platelet-rich plasma (PRP) [10] through interaction between LMW-H molecules in LMWH/P MPs'NPs and those GFs. LMW-H/P MPs-NPs are able to protect those GFs from heat and proteolytic inactivation and to enhance those biological activities. Since those GFs-containing LMW-H/P MPs-NPs are $200 \mathrm{~nm}$ $3 \mu \mathrm{m}$ in diameter, those can be easily injected [8-10]. Moreover, the GFs-containing the LMW-H/P MPs-NPs showed a substantial effect to induce vascularization and fibrous tissue formations due to stabilizating, activating, and gradually releasing GFs molecules from GFs-containing the LMW-H/P MPs·NPs [8-10].

In this review articles, we described on the LMW-H/P MPs-NPs which we originally prepared as PECs, its characterizations and its potential medical applications as carriers for GFs such as FGF-2 and GFs in PRP. Furthermore, as a coating matrix, LMW-H/P MPs-NPs were efficiently bound to tissue culture plates. With the ability of LMW-H/P MPs-NPs to retain GFs, the LMW-H/P MPs.NPs could serve as a useful coating matrix for culture of various types of cells.

\section{Preparation of LMW-H/P MPs·NPs}

Heparin interacts with a variety of functional proteins, including heparin-binding growth factors (GFs), cytokines, extracellular matrix components, and adhesion molecules [11-13]. Thus, heparin may be useful as a therapeutic agent in various pathological conditions that involve functional proteins. However, high-dose heparin cannot be used because of the excessive risk of bleeding [14]. In contrast, low-molecular-weight heparin (LMW$\mathrm{H}$, MW: approximately $5000 \mathrm{Da}$ ) which has much lower anti-coagulant activity, has pharmacological and practical advantages compared with native heparin. The lower protein binding activity of LMW-H produces a low, stable, and predictable anticoagulant response, thereby bypassing the need for laboratory monitoring of drug levels to adjust the dosage [14]. In addition, one or two subcutaneous injections per day are sufficient to maintain therapeutic concentrations because of its longer plasma half-life [14].

On the other hand, protamine, a purified mixture of proteins obtained from fish sperm, neutralizes heparin and LMW-H by forming a stable complex that lacks anticoagulant activity [15]. Protamine is also in clinical use to reverse the anticoagulant activity of heparin following cardiopulmonary bypass as well as in cases of heparininduced bleeding [16]. We previously prepared waterinsoluble particles $(>10 \mu \mathrm{m}$ in diameter) by mixing nonanticoagulant heparin with chitosan. We then mixed fucoidan with chitosan and investigated the ability of the resulting insoluble fucoidan/chitosan microparticles to protect fibroblast growth factor-2 (FGF-2) activity [17, $18]$. We also prepared water-insoluble micro (nano) particles $(200 \mathrm{~nm}-3 \mu \mathrm{m}$ in diameter) by mixing LMW-H $(6.4 \mathrm{mg} / \mathrm{ml})$ with protamine $(10 \mathrm{mg} / \mathrm{ml})$ at a ratio of $7: 3$ (vol:vol), and reported the ability of the resulting injectable low-molecular-weight heparin/protamine micro (nano) particles (LMW-H/P MPs-NPs) to protect FGF-2 activity (see Figure 1) [8,9]. Furthermore, GFs released from platelets that were involved in cell proliferation, migration, and angiogenesis were able to adsorb onto LMW-H/P MPs'NPs [10].

In order to produce of the nanoparticles, equally diluted LMW-H and protamine (100-fold, 50-fold, and 20 -fold diluted) were mixed in a ratio at 7:3 (vol:vol) in this study (see Figure 2). The diameter of generated LMW-H/P NPs by mixing 100 -fold, 50 -fold, and 20 -fold diluted protamine to equally diluted LMW-H in the ratio of 3:7 (vol:vol) were $84.6 \pm 26.8,95.0 \pm 27.0$, and 112.5 $\pm 46.1 \mathrm{~nm}$, respectively (see Figure 2) [9]. And no microparticles $(>1 \mu \mathrm{m}$ in diameter) were observed in the mixtures. In contrast, generations of small amount of microparticles (approximately $1 \mu \mathrm{m}$ in diameter) were observed by mixing of 10 -fold diluted protamine (1 $\mathrm{mg} / \mathrm{ml})$ to PMW-H $(0.64 \mathrm{mg} / \mathrm{ml})$ in the ratio of $3: 7$ ( $\mathrm{vol} / \mathrm{vol})$. When non-diluted protamine $(10 \mathrm{mg} / \mathrm{ml})$ was added to non-diluted LMW-H $(6.4 \mathrm{mg} / \mathrm{ml})$ up to ratio of 3:7 (vol:vol), maximal LMW-H/P MPs (200 nm - $3 \mu \mathrm{m}$ in diameter) was produced and the high turbidity was observed. When 10-fold concentrated protamine (100 $\mathrm{mg} / \mathrm{ml}$ ) was added to the equally 10 -fold concentrated LMW-H $(64 \mathrm{mg} / \mathrm{ml})$ up to ratio of 3:7 (vol:vol), mixtures of larger LMW-H/P MPs (3 - $10 \mu \mathrm{m}$ in diameter) and larger cotton-like precipitates $(>10 \mu \mathrm{m})$ were immediately generated and those products were insoluble [9]. An illustration on generations of micro/nanoparticles and insoluble precipitates by mixing protamine to LMW-H in various concentrations was shown in Figure 3.

Cotton-like compounds were generated after lyophilizations of both LMW-H/P MPs.NPs (200 nm - $3 \mu \mathrm{m}$ in diameter) and LMW-H/P NPs (approximately $100 \mathrm{~nm}$ in diameter) solutions without dextran, and they were hardly re-soluble in water. However, both the freeze- 


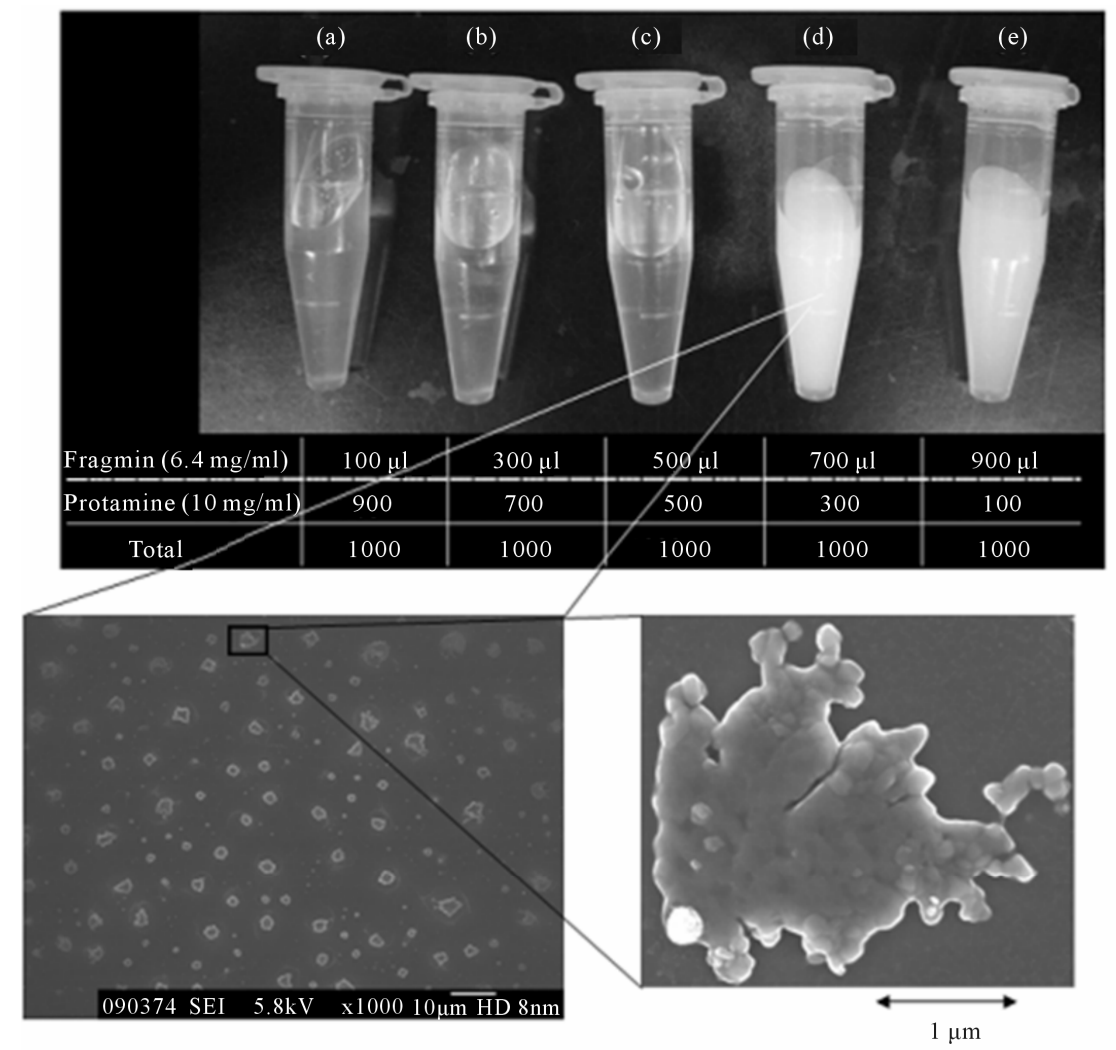

LMW-H/P MPs:NPs were prepared by mixing LMW-H and $P($ protamine $)$ with 7:3 (vol:vol)

Figure 1. Preparation of LMW-H/P MPs·NPs.

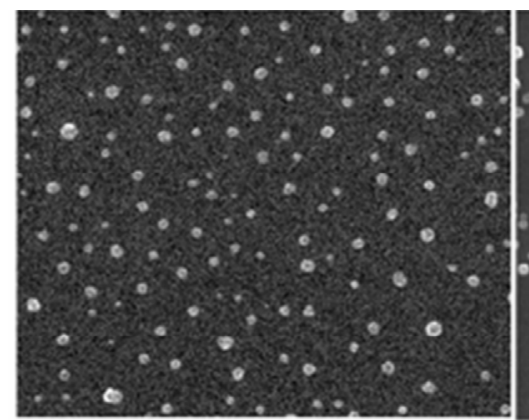

100-fold diluted

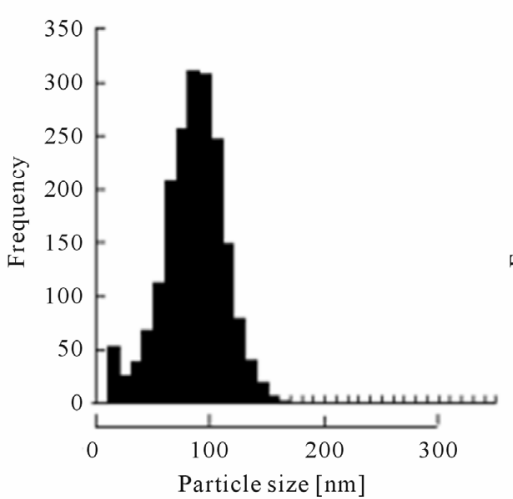

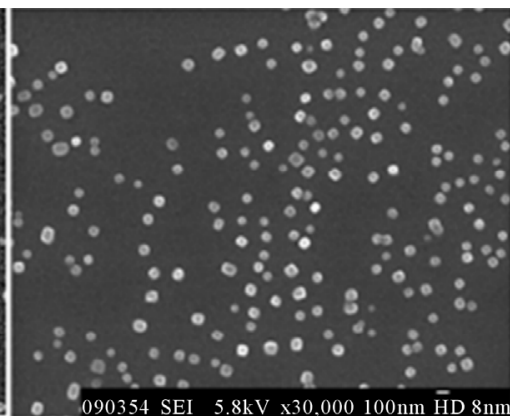

50-fold diluted

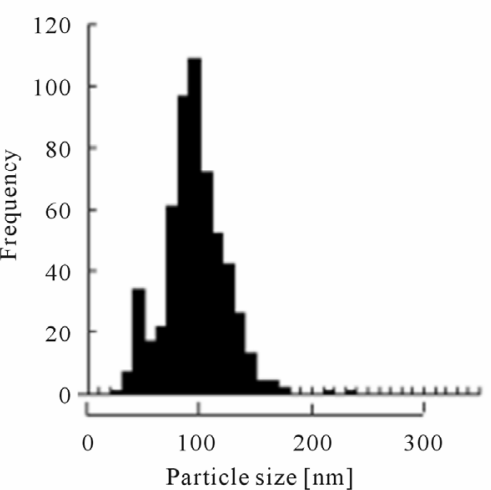

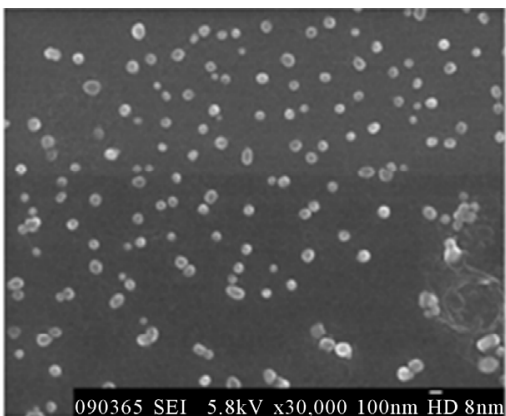

20-fold diluted

$1 \mu \mathrm{m}$

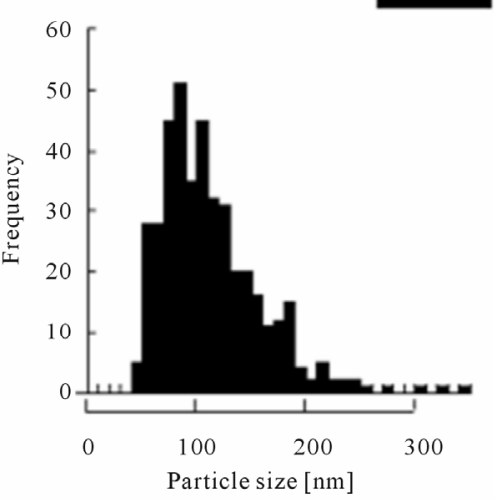

Figure 2. LMW-H/P NPs by mixing diluted protamine and LMW-H. 


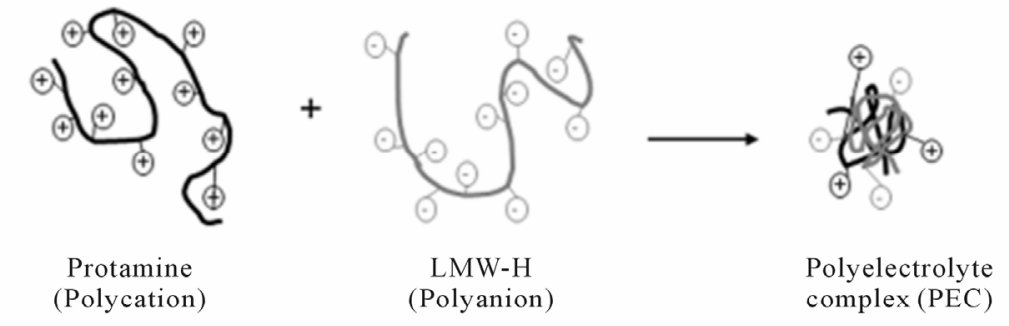

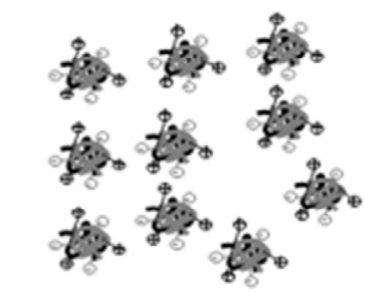

Soluble PECs ( $\leqq 100$ nm)

Nanoparticles

Low concentration

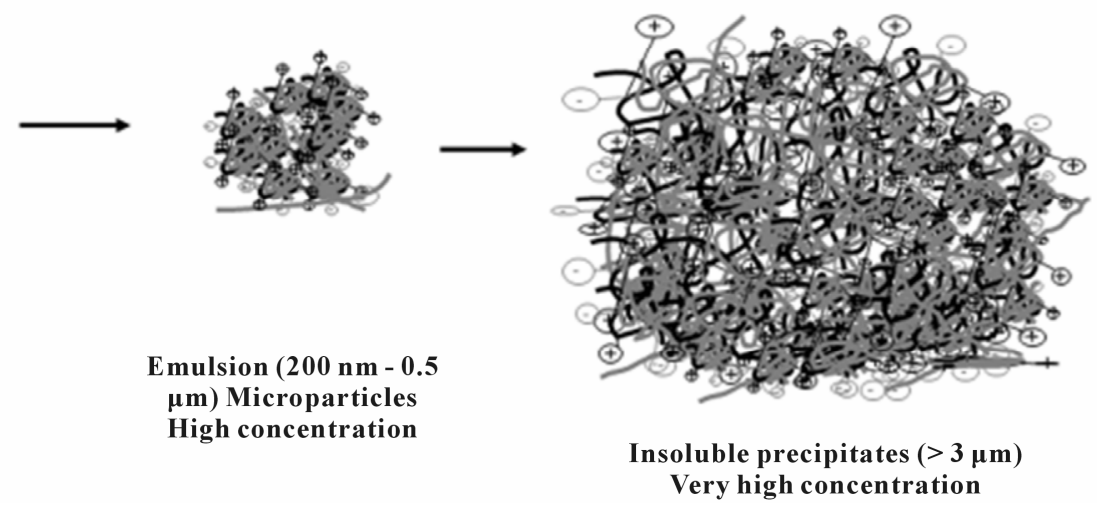

Figure 3. Generation of LMW-H/P MPs·NPs as PECs.

dried LMW-H/P MPs-NPs were easily dessolved in water by adding $0.5 \%$ and $0.2 \%$ dextran, respectively, before their lyophilizations. In addition, aggregation of LMW-H/P NPs in solution to LMW-H/P MPs was prohibited in the presence of dextran [9]. Thus, the addition of dextran is effective to stabilize the LMW-H/P MPs-NPs and to prepare stable and resoluble freeze-dry LMW-H/P MPs'NPs.

As a coating matrix, LMW-H/P MPs'NPs were efficiently bound to tissue culture plates. With the ability of LMW-H/P MPs-NPs to adsorb and retain GFs, the LMW-H/P MPs-NPs could be very useful in various types of cell culture as a coating matrix. Human microvascular endothelial cells and human dermal fibroblast cells well adhered to LMW-H/P MPs-NPs-coated tissue culture plates [19] and grew optimally in low fetal bovine serum (FBS) $(1 \%-2 \%)$ medium supplemented with FGF-2 $(5 \mathrm{ng} / \mathrm{ml})$. This protocol could make it possible to use low autologous human serum $(1 \%-2 \%)$ for the culturing of human bone marrow-derived mesenchymal stem cells (BMSCs) and human adipose-derived stromal cells (ASCs) [20]. Furthermore, CD34+ hematopoietic progenitor cells $(\mathrm{CD} 34+$ cells) derived from human bone marrow exhibited a comparatively higher proliferation on LMW-H/P MPs-NPs-coated plates in hematopoietic progenitor growth medium (HPGM) supplemented with appropriate cytokines than those on uncoated plates [21].

\section{Protein-Delivery Micro (Nano) Particles}

\subsection{FGF-2 Containing LMW-H/P MPs-NPs}

FGF-2 binds heparin with high affinity (Kd of $8.6 \times 10^{-9}$ $\mathrm{M})$. The polysaccharides can prolong the biological halflife of FGF-2 as well as protect FGF-2 from heat, acid, and proteolytic inactivation [22]. Similarly, the LMWH/P MPs:NPs have high affinity for FGF-2 $(\mathrm{Kd}=2.4 \times$ $\left.10^{-9} \mathrm{M}\right)$ [8], and this interaction of FGF-2 with the LMW-H/P MPs'NPs can substantially prolong the biological half-life time of FGF-2. The protection of FGF-2 against heat inactivation and trypsin degradation by the LMW-H/P MPs was effective in a concentration-dependent manner [8]. FGF-2 molecules were released in vitro from the FGF-2-containing LMW-H/P MPs-NPs with half-releasing time of about 6 days. Those results demonstrated that FGF-2 molecules are bound and stabilized on the LMW-H/P MPs-NPs, and that the FGF-2 molecules incorporated into the LMW-H/P MPs.NPs will be gradually released upon biodegradation of the hydrogel in vivo.

When the FGF-2-containing LMW-H/P MPs-NPs were subcutaneously injected into the backs of mice, neovascularization was induced near the injection site after 3 days. Neovascularization induced by the FGF-2-containing LMW-H/P MPs-NPs reached a maximum at 1 week, after which a slight decrease in the neovascularization rate occurred. No significant vascularization was 
observed after either the injection of FGF-2 alone or the LMW-H/P MPs'NPs alone [8].

Another study demonstrated advanced fat survival and capillary formation in FGF-2-containing LMW-H/P MPs-NPs-assist subdivided free fat-grafting groups in rats [23]. Furthermore, our study demonstrated the ability of FGF-2-containing LMW-H/P MPs-NPs to induce both arteriogenesis and angiogenesis in rabbit models of ischemic limbs (see Figure 4) [24]. The primary conclusion is that FGF-2-containing LMW-H/P MPs-NPs-treatment effectively induces the development of collateral vessels, which can provide sufficient blood flow to the pre-existing vascular network in ischemic tissue. Since all components used in the FGF-2-containing LMW-H/P MPs-NPs are also used clinically, we feel safety in a clinical setting is probable [24].

\subsection{Platelet-Rich Plasma (PRP) Containing LMW-H/P MPs·NPs}

PRP contains a high concentration of thrombocytes (platelets). When the platelets are activated, various GFs and other bioactive proteins in $\alpha$-granules of platelets are released and those proteins augment tissue repair and regeneration processes $[10,25,26]$. Platelets contain over $20 \mathrm{GFs}$, including platelet-derived growth factors (PDGFs),
FGFs, hepatocyte growth factor (HGF), transforming growth factors (TGFs), and vascular endothelial growth factors (VEGFs), almost all of which are known to bind to heparin and to LMW-H/P MPs-NPs. Recent studies suggest that GFs in PRP not only influence the viability of transferred cells but may also play bioactive roles in the regulation of proliferation and differentiation in various types of cells [10]. Any treatment aiming to mimic the critical aspects of the natural biological process should not be limited to the provision of a single GF, but rather should release multiple GFs at an optimized ratio, at a physiological dose and in a specific spatiotemporal pattern. Those results indicated that the LMW$\mathrm{H} / \mathrm{P}$ MPs.NPs also activate the platelets to release the GFs, and that in turn the released GFs from the platelets can be immobilized, be stabilized, and be activated on the LMW-H/P MPs.NPs [10].

The GFs in PRP are stably bound to LMW-H/P MPs in vivo. The GFs adsorbed onto LMW-H/P MPs-NPs may be gradually diffused and released upon biodegradation of LMW-H/P MPs.NPs. When PRP-containing LMW-H/P MPs-NPs were subcutaneously injected into the backs of mice, significantly higher neovascularization and granulation tissue with enhanced filtration of inflammatory cells were observed compared with the mouse
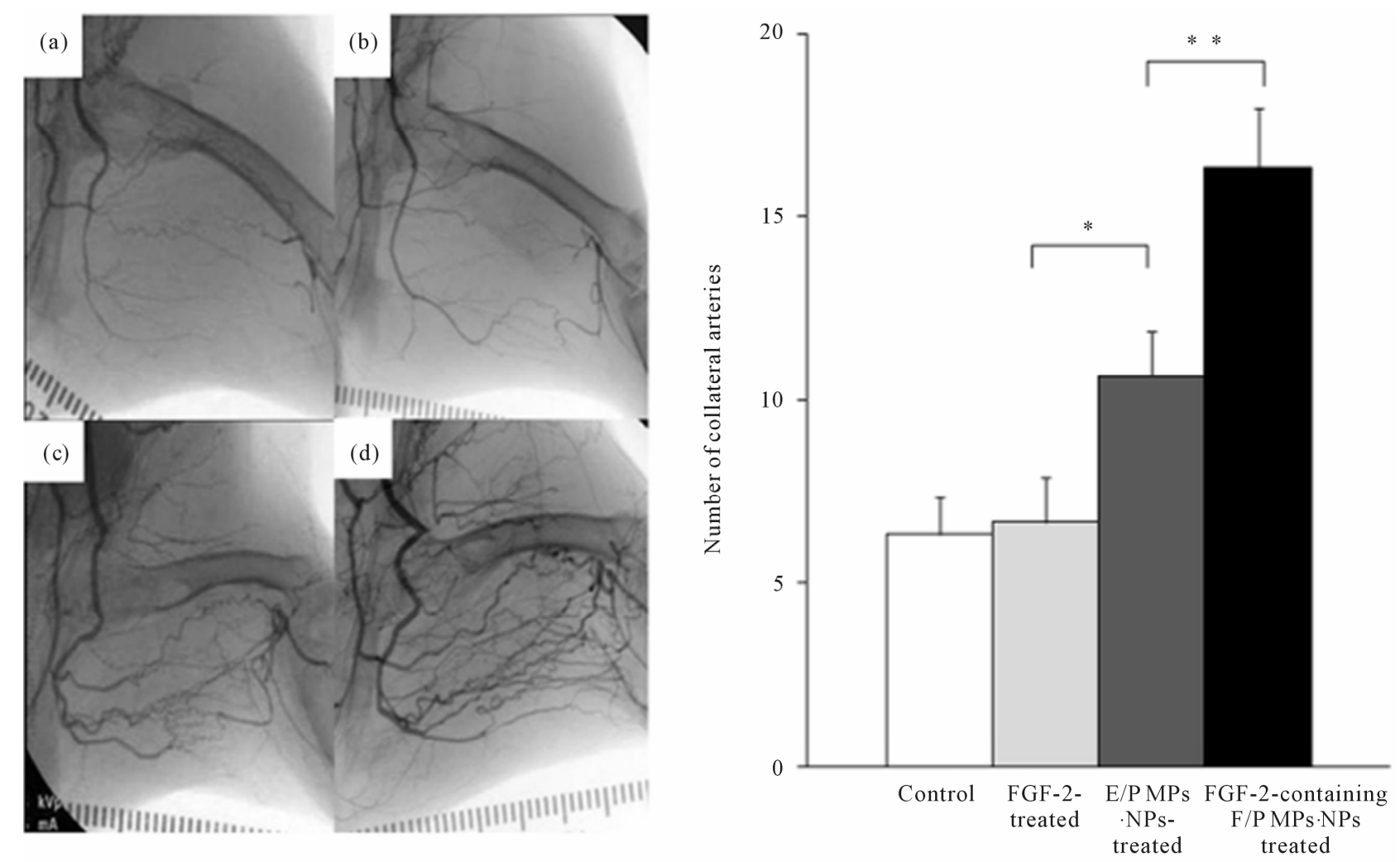

Figure 4. Quantification of visible collateral arteries under angiographic viewing on day 28. 
groups injected with PRP alone, LMW-H/P MPs-NPs alone, and the control [18]. Compared to either PRP alone or LMW-H/P MPs-NPs alone, locally administered PRP-containing LMW-H/P MPs-NPs augmented the wound bed and substantially increased viability of rat dorsal paired pedicle skin flaps [27]. The improved flap survival was noted if PRP-containing LMW-H/P MPs-NPs was administered 2 days before the flap elevation [27]. PRP-containing LMW-H/P MPs-NPs may thus represent a promising new biomaterial for improving skin flaps, particularly in the field of reconstructive surgery.

Clinical research was performed using autologous PRP-containing LMW-H/P MPs-NPs and PRP alone in 26 patients with thin hair (including 10 women) [28]. Hair growth and thickening following administration of both PRP-containing LMW-H/P MPs-NPs and PRP alone was observed in all patients compared with the control, but PRP-containing LMW-H/P MPs-NPs appeared to provide the most substantial change in the hair (see Figure 5) [28]. Because of the use of autologous materials, this method using PRP-containing LMW-H/P MPs-NPs is simpler, cheaper, and has no side effect compared with conventional methods.

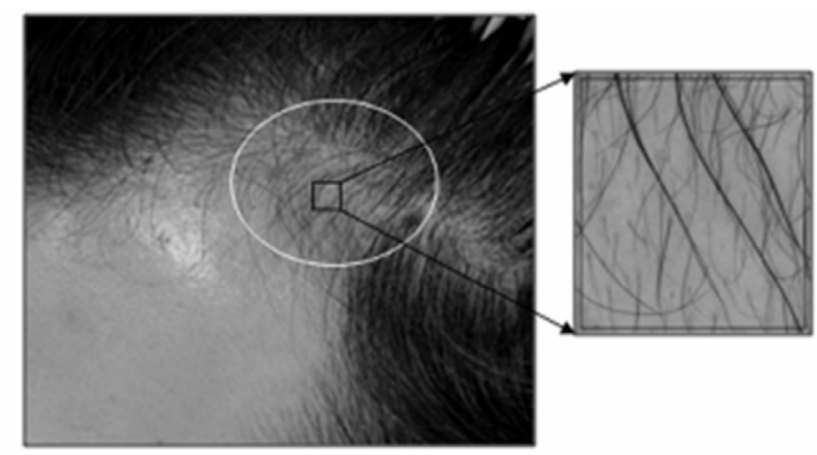

Before PRP-containing LMW-H/P MPs.NPs treatment.

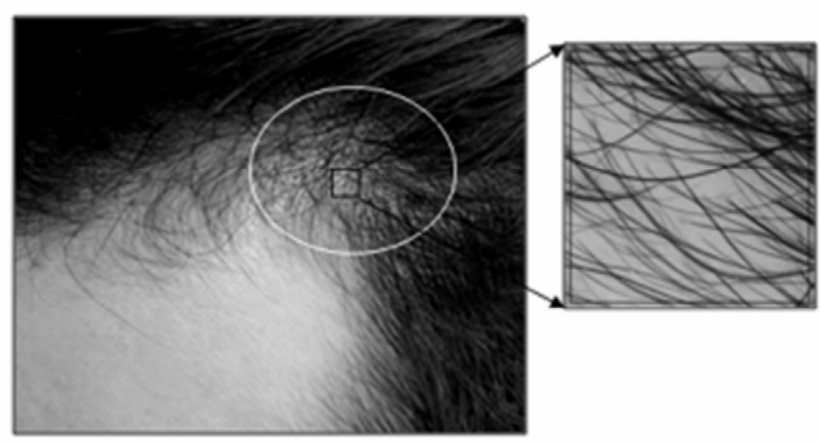

After PRP-containing LMW-H/P MPs-NPs treatment. (5 injections: 16 weeks)

\section{Cell Culture System Using Microparticle-Coated Plates}

\subsection{Various Types of Cell Cultures Using LMW-H/P MPs·NPs-Coated Plates}

The LMW-H/P MPs-NPs are able to attach to polymeric surfaces such as plastic and glass. The LMW-H/P MPs-NPs generate a stable paste-like coating through complete drying. It is probable that polypeptides, such as FGF-2, interleukin (IL)-3, and granulocyte/macrophagecolony stimulating factor (GM-CSF) once bound to the LMW-H/P MPs-NPs-coated plates, are gradually released from the coated surface in vitro with a half-life of 4 - 6 days [19]. Furthermore, LMW-H/P MPs-NPs- coating could optimally stimulate growth of human micro-vascular endothelial cells (hMVECs) and human dermal fibroblast cells (hDFCs) in low FBS $(1 \%-2 \%)$ DMEM with FGF-2 and growth of hematopoietic cell line (TF-1) with IL-3 and GM-CSF (see Figure 6) [19].

Heparin and heparinoids bind various GFs and cytokines including FGFs, HGF, VEGF, heparin-binding epidermal growth factor (HBEGF), PDGF, TGF- $\beta$, GM-CSF, interleukins (i.e., IL-1, IL-2, IL-3, IL-4, IL-6,

Hair cross-sections

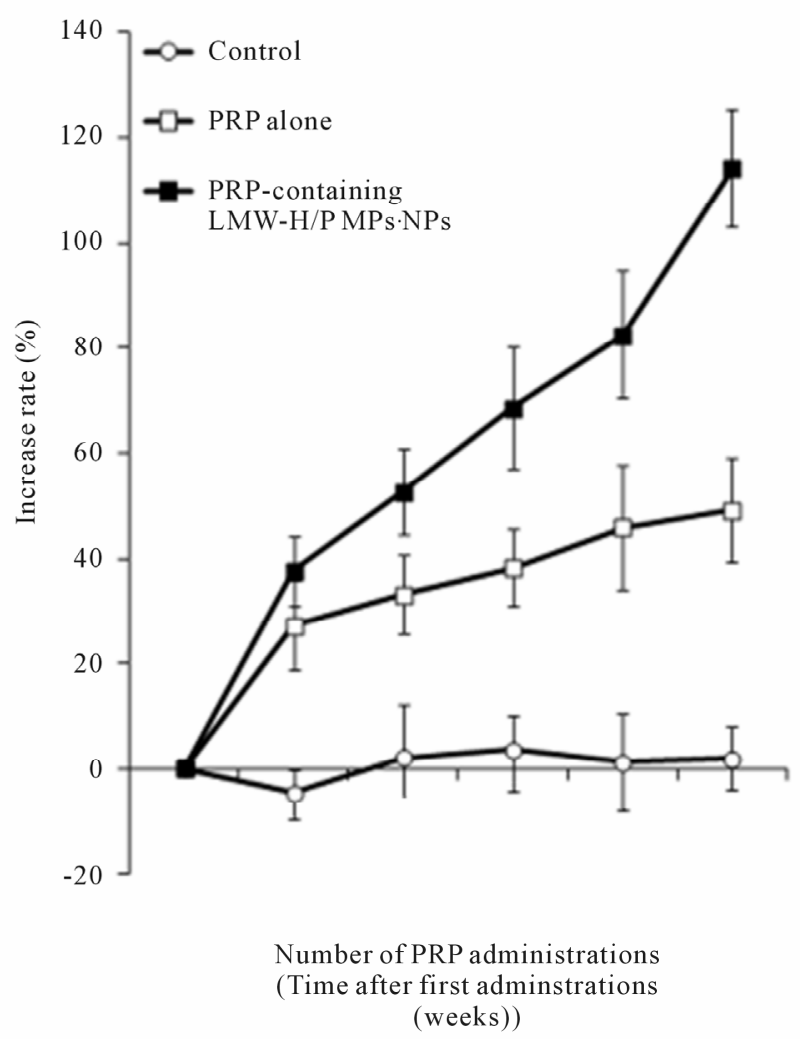

Figure 5. PRP-containing LMW-H/P MPs·NPs treatment for Alopecia Areata. 

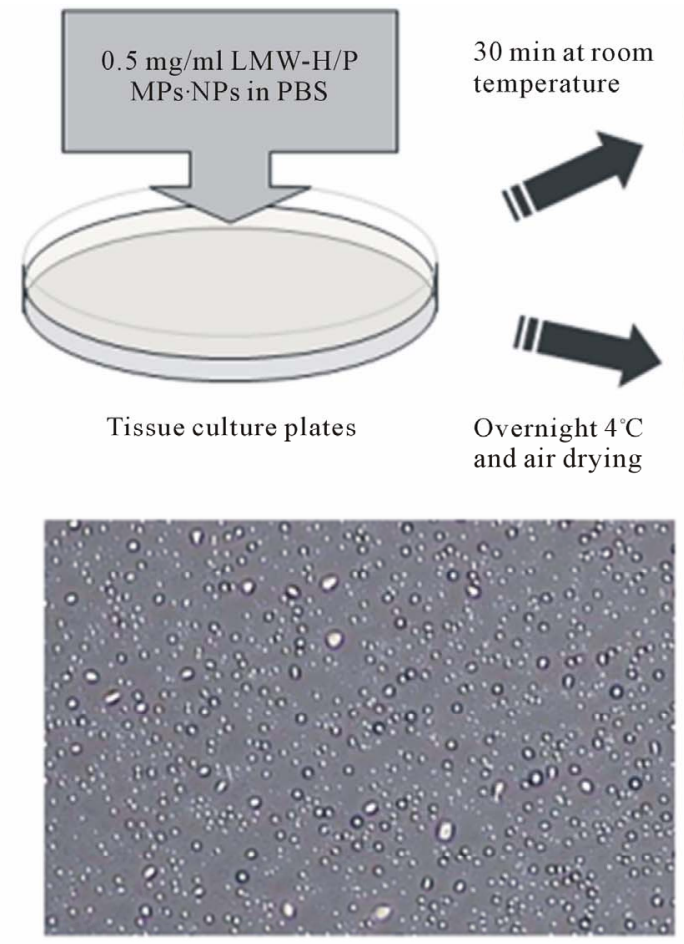

Low dense LMW-H/P MPs.NPs-coated plates

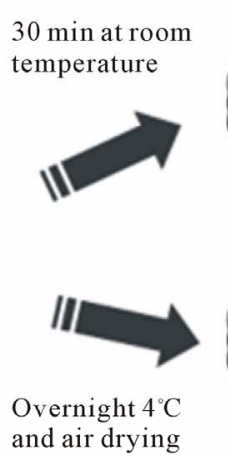

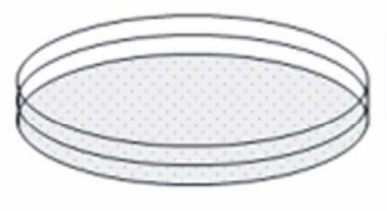
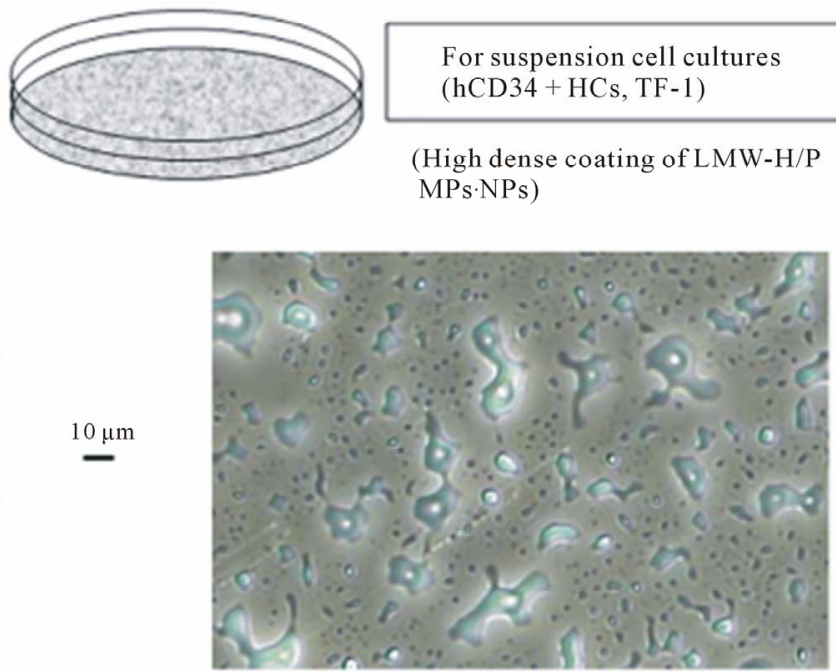

High dense LMW-H/P MPs-NPs-coated plates

Figure 6. Preparation of LMW-H/P MPs·NPs-coated plates.

IL-7, and IL-8), interferon $\gamma$, and macrophage inflammatory protein-1 [11-13]. These GFs and cytokines can potentially be immobilized on the LMW-H/P MPs-NPscoated plates. Actually, in addition to FGF-2, IL-3 and GM-CSF described above, we have already observed that FGF-1, HGF, HBEGF, TGF- $\beta$, human stem cell factor (SCF), thrombopoietin (Tpo), and Flt-3 ligand (Flt-3) could be efficiently immobilized on the LMW-H/P MPs-NPs-coated plates [21]. Furthermore, the bound GFs to the LMW-H/P MPs-NPs-coated plates appeared to enhance and to stabilize those biological activities. Thus, LMW-H/P MPs-NPs-coating provides an excellent biomaterial to immobilize and retain GFs and cytokines for optimal growth of various types of cells with low (no) serum medium (see Figure 6).

\subsection{Proliferation of BMSCs and ASCs on LMW-H/P MPs·NPs-Coated Plates}

Cell-based therapies such as tissue engineering will benefit from a source of autologous multipotent stem cells, including bone marrow-derived mesenchymal stem cells (BMSCs) and adipose tissue-derived stromal cells (ASCs). There are two stem cell lineages in bone marrow cell populations, i.e., hematopoietic cells ( $\mathrm{HCs}$ ) and BMSCs. The BMSCs and ASCs are multipotential, indicating that in culture $[29,30]$ or after in vivo implantation these cells can differentiate into a variety of cell types including osteoblasts, chondrocytes, adipocytes, myoblasts [31], and neuronal cells [32]. Furthermore, cultured ASCs secreted significant amounts of angiogenic growth factors such as FGF-2, HGF, PDGF, and VEGF at levels that are bioactive [33]. Thus, LMW-H/P MPs-NPs may serve as an effective matrix for cultures of BMSCs and ASCs. The safe and effective expansions of BMSCs and ASCs represent a promising option for tissue engineering strategies.

Most protocols for the expansion of BMSCs and ASCs include high concentrations $(10 \%-20 \%)$ of animal serum such as FBS as a nutritional supplement. In some cell cultures, this involves multiple doses of FBS, which raises concerns over possible contamination as well as immunological reactions caused by medium-derived FBS proteins, sialic acid derivatives, etc. [34,35]. Patients may experience problems when undergoing autologous cell-based therapies if a serum other than an autologous serum is used during the culturing of the cells. However, it would be difficult to obtain large amounts of autologous serum from the patient for large-scale autologous cell culture [20]. It should be noted that the growth of cultured BMSCs or ASCs on LMW-H/P MPs-NP-coated plates in combination with FGF-2 and FBS (1\%-2\%) was significantly stimulated, and similar stimulation was 
observed in those cultured cells on LMW-H/P MPs'NPscoated plates with FGF-2 and 1\% - 2\% human serum (HS) prepared from adult bloods instead of FBS.

\subsection{Proliferation of CD34+ Hematopoietic Progenitor Cells (CD34+ HCs) on LMW-H/P MPs:NPs-Coated Plates}

Hematopoietic progenitor cells proliferate and mature in semi-solid media when stimulated by exogenous hematopoietic cell growth factors (HCGFs) such as SCF, Tpo, Flt-3, IL-3, and GM-CSF [36,37]. These cells also proliferate in association with bone marrow-derived stromal cells (BMSCs) [38,39], although biologically active amounts of HCGFs cannot be detected in stromal culture supernatants $[38,39]$. It is possible that HCGFs are synthesized by the stromal cells but remain bound to the stromal cells and/or their extracellular matrix. In fact, it was demonstrated that both natural and recombinant HCGFs, such as IL-3 and GM-CSF, could be adsorbed by heparan sulfate, which is the major sulfated glycosaminoglycan of bone marrow stroma $[38,39]$. Serum-free medium supplemented with large amounts of SCF, Tpo, and Flt-3 was reported for expansion of CD34+ HCs [40, 41]. Although such medium is commercially avail- able (HPGM, Lonza Japan Corp., Tokyo, Japan), it is prohibitively expensive. We demonstrated that recombinant HCGFs such as SCF, Tpo, and Flt-3 were immobilized onto LMW-H/P MPs-NPs-coated plates, and the immobilized cytokines were stabilized, were activated, and were gradually released into the medium. Those cytokines, once bound, can be presented in the biologically active form to hematopoietic progenitor cells $[38,39]$. Furthermore, only one-fourth of the concentration of the cytokines recommended by the manufacture was required for maximal expansion of CD34+ HCs on the LMW-H/P MPs-NPs-coated plates. These findings may have important implications for the use of heparinoid as an artificial matrix for ex vivo expansion of hematopoietic progenitor cells with adequate cytokines. The LMW-H/P MPs-NPscoating matrix in the presence of lower concentrations of SCF, Tpo, and Flt-3 is a convenient and safe material for stable expansion of CD34+ HCs using HPGM without any animal serum.

\section{Conclusions}

It is recognized in polymer chemistry that positively and negatively charged polymers interact ionically [17]. Through these ionic interactions, basic protamine molecules can bind with acidic molecules (LMW-H) to form micro (nano) particle complexes. We previously reported that GF-containing LMW-H/P MPs-NPs, which are 200 $\mathrm{nm}-3 \mu \mathrm{m}$ in diameter, can be easily injected [8-10]. Fur- thermore, the LMW-H/P MPs-NPs were observed on the protection of FGF-2 and GFs in PRP activity from heat and proteolytic inactivation. These results indicate that LMW-H/P MPs-NPs may serve as an effective microcarrier for various GFs, particularly for the local application of GFs. GFs-containing LMW-H/P MPs-NPs show a substantial effect to induce vascularization and fibrous tissue formation because of stabilization, activation, and gradual release of GF molecules from GFs-containing LMW-H/P MPs-NPs [8-10].

The presented method for the optimal proliferation and differentiation of ASCs and BMSCs on LMW-H/P MPs.NPs-coated plates in low concentration human serum medium (1\% - 2\%) supplemented with FGF-2 (5 $\mathrm{ng} / \mathrm{ml}$ ). No animal serum is required in the culture of those cell types. The bound GFs to the LMW-H/P MPs-NPs-coated plates appeared to enhance and to stabilize those biological activities. The proliferated cells maintained their potential to differentiate into adipocytes and osteoblasts [20,33]. Furthermore, the LMW-H/P MPs-NPs-coating matrix in the presence of lower concentrations of SCF, Tpo, and Flt-3 were convenient materials for stable expansion of CD34+ HCs using HPGM without any animal serum. These results suggest a promising cell source, particularly for the preparation of large amounts of ASCs, BMSCs, or CD34+ HCs required for cell-based therapies in several clinical fields.

LMW-H, protamine, several GFs and cytokines, and autologous PRP are already in clinical use. Since autologous ASCs, BMSCs, or CD34+ HCs are available, the clinical safety of LMW-H/P MPs-NPs as protein-carrier is possible. Furthermore, ASCs, BMSCs, or CD34+ HCs can be efficiently expanded as cell sources for regenerative medicines with the use of LMW-H/P MPs-NPscoated plates as a matrix without animal serum or feeder cells.

\section{REFERENCES}

[1] J. M. Park, B. B. Muhoberac, P. L Dubin and J. Xia, "Effect of Protein Charge Heterogeneity in Protein-Polyelectrolyte Complexation," Macromolecules, Vol. 25, No. 1, 1992, pp. 290-295. doi:10.1021/ma00027a047

[2] K. W. Mattison, P. L. Dubin and I. J. Brittain, "Complex Formation between Bovine Serum Albumin and Strong Polyelectrolytes: Effect of Polymer Charge Density," Journal of Physical Chemistry B, Vol. 102, No. 19, 1998, pp. 3830-3836. doi:10.1021/jp980486u

[3] S. Dragan, M. Cristea, C. Luca and B. C. Simionescu, "Polyelectrolyte Complex. I: Synthesis and Characterization of Some Insoluble Polyanion-Polycation Complexes," Journal of Polymer Science A, Vol. 34, No. 17, 1996, pp. 3487-3495. doi:10.1002/(SICI)1099-0518(199612)34:17<3485::AID- 


\section{POLA3>3.0.CO;2-U}

[4] Y. Koyama, M. Yamashita, N. I. Tanaka and T. Ito, "Enhancement of Transcriptional Activity of DNA Complexes by Amphoteric PEG Derivertive," Biomacromolecules, Vol. 7, No. 4, 2006, pp. 1274-1279. doi: $10.1021 / \mathrm{bm} 0504633$

[5] L. Webster, M. B. Huglin and I. D. Robb, "Complex Formation between Poly-Electrolytes in Dilute Aqueous Solution," Polymer, Vol. 38, No. 6, 1997, pp. 1373-1380. doi:10.1016/S0032-3861(96)00650-7

[6] M. Hashimoto, Y. Koyama and T. Sato, "In Vitro Gene Delivery by pDNA/Chitosan Complexes Coated with Anionic PEG Derivatives That Have a Sugar Side Chain," Chemistry Letter, Vol. 37, No. 3, 2008, pp. 266-267. doi:10.1246/cl.2008.266

[7] A. Denuziere, D. Ferrier and A. Domard, "ChitosanChondroitin Sulfate and Chitosan-Hyaluronate Polyelectrolyte Complexes. Physico-Chemical Aspects," Carbohydrate Polymer, Vol. 29, No. 4, 1996, pp. 317-323. doi:10.1016/S0144-8617(96)00035-5

[8] S. Nakamura, Y. Kanatani, S. Kishimoto, M. Nambu, C. Ohno, H. Hattori, B. Takase, Y. Tanaka, H. Yura, T. Kiyosawa, T. Maehara and M. Ishihara, "Controlled Release of FGF-2 Using Fragmin/Protamine Microparticles and Effect on Neovascularization," Journal of Biomedical Materials Research A, Vol. 91, No. 3, 2009, pp. 814-823. doi:10.1002/jbm.a.32265

[9] Y. Mori, S. Nakamura, S. Kishimoto, M. Kawakami, S. Satoshi, T. Matsui and M. Ishihara, "Preparation and Characterization of Low-Molecular-Weight Heparin/Protamine Nanoparticles (LMW-H/P NPs) as FGF-2 Carrier," International Journal of Nanomedicine, Vol. 5, 2010, pp. 147-155. doi:10.2147/IJN.S8692

[10] M. Takikawa, S.-I. Nakamura, S. Nakamura, M. Nambu, M. Ishihara, M. Fujita, S. Kishimoto, T. Doumoto, S. Yanagibayashi, R. Azuma, N. Yamamoto and T. Kiyosawa, "Enhancement of Vascularization and Granulation Tissue Formation by Growth Factors in Human PlateletRich Plasma-Containing Fragmin/Protamine Microparticles," Journal of Biomedical Materials Research B, Vol. 97, 2011, pp. 373-380. doi:10.1002/jbm.b.31824

[11] M. Ishihara and K. Ono, "Structure and Function of Heparin and Heparan Sulfate: Heparinoid Library and Modification of FGF-Activities," Trends in Glycoscience and Glycotechnoogy, Vol. 10, No. 52, 1998, pp. 223-233. doi:10.4052/tigg. 10.223

[12] M. Salmivirta, K. Lidhold and U. Lindahl, "Heparan Sulfate: A Piece of Information," FASEB Journal, Vol. 10, No. 52, 1996, pp. 1270-1279.

[13] U. Lindahl, K. Lidholt, D. Spillmann and L. Kjellen, "More to 'Heparin' Than Anti-Coagulation," Thrombosis Research, Vol. 75, No. 1, 1994, pp. 1-32. doi:10.1016/0049-3848(94)90136-8

[14] J. Hirsh, T. E. Warkentin, S. G. Shaughnessy, S. S. Anand, J. L. Halperin, R. Raschke and C. Granger, "Heparin and Low-Molecular Heparin, Mechanisms of Action,
Phormacokinetics, Dosing, Monitoring, Efficacy, and Safety," Chest, Vol. 119, No. 2, 2001, pp. 64-94. doi:10.1378/chest.119.1_suppl.645

[15] M. Wolzt, A. Wetermann, M. Nieszpaur-Los, B. Schneider, A. Fassolt, K. Lechner, H. Eichler and P. A. Kyrle, "Studies on the Neutralizing Effects of Protamine on Unfractionated and Low Molecular Weight Heparin (Frag$\min ())$ at the Site of Activation of the Coagulation System in Man," Thrombosis and Haemostasis, Vol. 73, No. 3, 1995, pp. 439-443.

[16] M. Pan, J. S. Lezo, A. Medina, M. Romero, E. Hernandez, J. Segura, F. Melian, F. Wanguemert, M. Landin, F. Benitez, M. Amay, F. Velasco and A. Torres, "In-Laboratory Removal of Femoral Sheath Following Protamine Administration in Patients Having Intracoronary Stent Implantation," American Journal of Cardiology, Vol. 80, No. 10, 1997, pp. 1336-1338. doi:10.1016/S0002-9149(97)00676-0

[17] M. Fujita, M. Ishihara, M. Shimizu, K. Obara, T. Ishizuka, Y. Saito, H. Yura, Y. Morimoto, B. Takase, T. Matsui, M. Kikuchi and A. Kurita, "Vascularization in Vivo Caused by the Controlled Release of Fibroblast Growth Factor-2 from an Injectable Chitosan/Non-Anticoagulant Heparin Hydrogel," Biomaterials, Vol. 25, No. 4, 2004, pp. 699706. doi:10.1016/S0142-9612(03)00557-X

[18] S. Nakamura, M. Nambu, S. Kishimoto, T. Ishizuka, H. Hattori, Y. Kanatani, B. Takase, H. Aoki, T. Kiyosawa, T. Maehara and M. Ishihara, "Effect of Controlled Release of Fibroblast Growth Factor-2 from Chitosan/Fucoidan Micro Complex Hydrogel on in Vitro and in Vivo Vascularization," Journal of Biomedical Materials Research A, Vol. 85, 2008, pp. 619-627. doi:10.1002/jbm.a.31563

[19] S. Kishimoto, S. Nakamura, S.-I. Nakamura, Y. Kanatani, H. Hattori, Y. Tanaka, Y. Harada, M. Tagawa, Y. Mori, T. Maehara and M. Ishihara, "Fragmin/protamine Microparticle-Coated Matrix Immobilized Cytokines to Stimulate Various Cell Proliferations with Low Serum Media," Artificial Organs, Vol. 33, No. 6, 2009, pp. 431-438. doi:10.1111/j.1525-1594.2009.00745.x

[20] S. Kishimoto, H. Hattori, S. Nakamura, Y. Amano, Y. Kanatani, Y. Tanaka, Y. Mori, Y. Harada, M. Tagawa and M. Ishihara, "Expansion and Characterization of Human bone Marrow-Derived Mesenchymal Stem Cells Cultured on Fragmin/Protamine Microparticle-Coated Matrix with Fibroblast Growth Factor-2 in Low Serum Medium," Tissue Engineering Part C, Vol. 15, No. 3, 2009, pp. 523- 527. doi:10.1089/ten.tec.2008.0492

[21] S. Kishimoto, S. Nakamura, S.-I. Nakamura, H. Hattori, F. Oomuma, Y. Kanatani, Y. Tanaka, Y. Harada, M. Tagawa, T. Maehara and M. Ishihara, "Cytokine-Immobilized Microparticle-Coated Plates for Culturing Hematopoietic Progenitor Cells," Journal of Controlled Release, Vol. 133, 2009, pp. 185-190. doi: $10.1016 / \mathrm{j} / \mathrm{jconrel}, 2008.10 .005$

[22] D. Gospodarowicz and J. Cheng, "Heparin Protects Basic and Acidic FGF from Inactivation," Journal of Cellular Physiology, Vol. 128, No. 3, 1986, pp. 475-484. 


\section{doi:10.1002/jep.1041280317}

[23] S.-I. Nakamura, M. Ishihara, M. Takikawa, K. Murakami, S. Kishimoto, S. Nakamura, S. Yanagibayashi, Y. Mori, M. Fujita, S. Kubo, N. Yamamoto and T. Kiyosawa, "Increased Survival of Free Fat Grafts and Vascularization in Rats with Local Delivery of Fragmin/Protamine Microparticles Containing FGF-2 (F/P MP-F)," Journal of Biomedical Materials Research B, Vol. 96, 2011, pp. 234-241. doi:10.1002/jbm.b.31757

[24] T. Horio, M. Fujita, Y. Tanaka, M. Ishihara, S. Kishimoto, S. Nakamura, M. Shimizu, Y. Nogami, H. Hattori, K. Hase and T. Maehara, "Efficacy of Fragmin/Protamine Microparticles Containing Fibroblast Growth Factor-2 (F/P MP/FGF-2) in a Rabbit Model of Hindlimb Ischemia," Journal of Vascular Surgery, Vol. 54, 2011, pp. 791-798. doi:10.1016/j.jvs.2011.02.060

[25] R. E. Marx, "Platelet-Rich Plasma: Evidence to Support Its Use," Journal of Oral and Maxillofacial Surgery, Vol. 62, 2001, pp. 225-228. doi:10.1016/j.joms.2003.12.003

[26] B. L. Eppley, W. S. Pietrzak and M. Blanton, "Plateletrich Plasma: A Review of Biology and Application in Plastic Surgery," Plastic and Reconstructive Surgery, Vol. 118, 2002, pp. 147-159. doi:10.1097/01.prs.0000239606.92676.cf

[27] M. Takikawa, Y. Sumi, M. Ishihara, S. Kishimoto, S. Nakamura, S. Yanagibayashi, H. Hattori, R. Azuma, N. Yamamoto and T. Kiyosawa, "PRP\&F/P MPs Improved Survival of Dorsal Paired Pedicle Skin Flaps in Rats," Journal of Surgical Research, Vol. 170, 2011, pp. 189196. doi:10.1016/j/jss.2011.05.051

[28] M. Takikawa, S.-I. Nakamura, S. Nakamura, M. Nambu, M. Ishihara, K. Murakami, S. Kishimoto, K. Sasaki, S. Yanagishita, R. Azuma, N. Yamamoto and T. Kiyosawa, "Enhanced Effect of Platelet-Rich Plasma Containing a New Carrier on Hair Growth," Dermatologic Surgery, Vol. 37, 2011, pp. 1-9. doi:10.1111/j.1524-4725.2011.02123.x

[29] D. J. Prockop, "Marrow Stromal Cells as Stem Cells for Nonhematopoietic Tissues," Science, Vol. 276, No. 5309, 1997, pp. 71-74. doi:10.1126/science.276.5309.71

[30] M. F. Pittenger, A. M. Mackay, S. C. Beck, R. K. Jaiswal, R. Douglas, J. D. Mosca, M. A. Moorman, D. W. Simonetti, S. Craig and D. R. Marshak, "Multilineage Potential of Adult Human Mesenchymal Stem Cells," Science, Vol. 284, No. 5411, 1999, pp. 143-147. doi:10.1126/science.284.5411.143

[31] G. Ferrari, G. Cusella-DeAngelis, M. Coletta, E. Paolucci, A. Stornaiuolo, G. Cossu and F. Mavilio, "Muscle Regeneration by Bone Marrow-Derived Myogenic Progenitors," Science, Vol. 279, No. 5356, 1998, pp. 1528-1530. doi:10.1126/science.279.5356.1528

[32] T. M. Coyne, A. J. Marcus, K. Reynold, I. B. Black and D. Woodbury, "Desparate Host Response and Donor Survival after the Transplantation of Mesenchymal or Neu- roectodermal Cells to the Intact Roden Brain," Transplantation, Vol. 84, No. 11, 2007, pp. 1507-1516. doi:10.1097/01.tp.0000288185.09601.4d

[33] S. Nakamura, S. Kishimoto, S.-I. Nakamura, M. Nambu, M. Fujita, Y. Tanaka, Y. Mori, M. Tagawa, T. Maehara and M. Ishihara, "Fragmin/Protamine Microparticles as Cell Carriers to Enhance Viability of Adipose-Derived Stromal Cells and Their Subsequent Effect on in Vivo Neovascularization," Journal of Biomedical Matererials Research A, Vol. 92, 2010, pp. 1614-1622. doi:10.1002/jbm.a.32506

[34] J. L. Spees, C. A. Gregory, H. Singh, H. A. Tucker, A. Peister, P. J. Lynch, S. C. Hsu, J. Smith and D. J. Prockop, "Internalized Antigens Must Be Removed to Prepare Hypoimmunogenic Mesenchymal Stem Cells for Cell and Gene Therapy," Molecular Therapy, Vol. 9, No. 5, 2004, pp. 747-756. doi:10.1016/j.ymthe.2004.02.012

[35] M. J. Martin, A. Muotri, F. Gage and A. Varki, "Human Embryonic Stem Cells Express an Immunogenic Nonhuman Sialic Acid," Nature Medicine, Vol. 11, No. 2, 2005, pp. 228-232.

[36] P. Gupta, T. R. Oegema, J. J. Brazil, A. Z. Dudek, A. Slungaard and C. M. Verfaillie, "Structurally Specific Heparan Sulfates Support Primitive Human Hematopoiesis by Formation of a Multimolecular Stem Cell Niche," Blood, Vol. 92, No. 12, 1998, pp. 4641-4651.

[37] M. Alvarez-Silva and R. Borojevic, "GM-CSF and IL-3 Activities in Schistosomal Liver Granulomas Are Controlled by Stroma-Associated Heparan Sulfate Proteoglycans," Journal of Leukocyte Biology, Vol. 59, No. 3, 1996, pp. 435-441.

[38] R. Roberts, J. Gallagher, E. Spooncer, T. D. Allen, F. Bloomfield and T. M. Dexter, "Heparan Sulfate Bound Growth Factors: A Mechanism for Stromal Cell Mediated Haemopoiesis," Nature, Vol. 332, No. 6162, 1988, pp. 376-378. doi:10.1038/332376a0

[39] M. Y. Gordon, G. P. Riley, S. M. Watt and M. F. Greaves, "Compartmentalization of a Haematopoietic Growth Factor (GM-CSF) by Glycosaminoglycans in the Bone Marrow Microenvironment," Nature, Vol. 326, No. 6111, 1987, pp. 403-405. doi:10.1038/326403a0

[40] A. L. Drayer, S. G. Olthof and E. Vellenga, "Mammalian Target of Rapamycin Is Required for Thrombopoietin-Induced Proliferation of Megakaryocyte Progenitors," Stem Cells, Vol. 24, No. 1, 2006, pp. 105-114. doi:10.1634/stemcells-2005-0062

[41] H. Schepers, A. T. Wierenga, D. V. Gasliga, B. J. Eggen, E. Vellenga and J. J. Schuringa, "Reintroduction of C/EBPalpha in Leukemic CD34+ Stem/Progenitor Cells Impairs Self-Renewal and Partially Restores Myelopoiesis," Blood, Vol. 110, No. 4, 2007, pp. 1317-1325. doi:10.1182/blood-2006-10-052175 